\title{
Atherogenic index of plasma is a novel predictor of non-alcoholic fatty liver disease in obese participants: a cross-sectional study
}

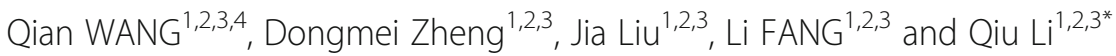

\begin{abstract}
Background: The atherogenic index of plasma showed to be related with some chronic disease like cardiovascular diseases and atherosclerosis. Body mass index which was commonly used in clinical practice is not an accurate index to predict non-alcoholic fatty liver disease. The aim of this study is to investigate the relationship between atherogenic index of plasma and non-alcoholic fatty liver disease in obese participants.

Methods: 538 obese subjects were included in this cross sectional study. Non-alcoholic fatty liver disease was diagnosed by B-ultrasonography after excluding participants with other liver diseases. The atherogenic index of plasma was classified into three groups: the low $(<0.11)$, the intermediate $(0.11-0.21)$ and the high $(>0.21)$ risk. The participants were separated into groups according to their atherogenic index of plasma levels. The area under receiver operating characteristic curve of the atherogenic index of plasma for predicting non-alcoholic fatty liver disease was calculated.

Results: There were concordances between increased atherogenic index of plasma and significant increase in the value of body mass index, waist circumference, alanine aminotransferase, glutamyl transpeptidase and lipid profile. The atherogenic index of plasma is strongly associated with non-alcoholic fatty liver disease. Compared to the low risk group, the high risk group had a 5.37 folds risk after adjustment for covariates. Results of receiver operating characteristic curves showed that the area under the curve (95\% confidence intervals) was $0.718(0.670-0.766)$.

Conclusion: These data suggest that atherogenic index of plasma might be a method which can be used in the auxiliary diagnosis of non-alcoholic fatty liver disease.
\end{abstract}

\section{Introduction}

Non-alcoholic fatty liver disease (NAFLD), initiated by the accumulation of fat in hepatocytes in the absence of excessive alcohol intake, is currently a common cause of chronic liver disease and has been associated with obesity, type 2 diabetes, hyperlipidemia, and insulin resistance. Throughout the world, the incidence rate of NAFLD is estimated to range from 5 to $30 \%$ depending

\footnotetext{
* Correspondence: liqiu10@163.com

${ }^{1}$ Department of Endocrinology, Shandong Provincial Hospital affiliated to Shandong University, 324 Jing 5 Road, Jinan 250021, Shandong Province, China

${ }^{2}$ Shandong Clinical Medical Center of Endocrinology and Metabolism, 324 Jing 5 Road, Jinan 250021, Shandong Province, China

Full list of author information is available at the end of the article
}

on geographical variations and its incidence rate has rapidly increased in recent decades [1, 2]. NAFLD which was considering as the new global epidemic needs better awareness.

With the prevalent of western diet, obesity becomes a more important medical and social problem. More than half of the people in the US were overweight or even worse. About $30.48 \%$ of the people were overweight, and $8.77 \%$ were obesity in China [3,4]. Overweight and obesity are well-established risk factors for diabetes, cardiovascular disease (CVD), certain cancers and premature death, as well as adverse reproductive consequences [5-7].

Although the fact that obesity increases risk of certain diseases is well accepted by clinicians, evidence has

(c) The Author(s). 2018 Open Access This article is distributed under the terms of the Creative Commons Attribution 4.0 International License (http://creativecommons.org/licenses/by/4.0/), which permits unrestricted use, distribution, and 
shown that some individuals who are obese are also metabolically healthy. Increasing evidence suggests that body mass index (BMI), the most common proxy measure used to help categorize overweight or obesity, is an imprecise measure of body-fat-related risk of non-communicable diseases.

The atherogenic index of plasma (AIP), the logarithm of the molar ratio of triglyceride to high density lipoprotein cholesterol (TG/HDL-C), has shown a strong correlation with size of the low density lipoprotein cholesterol (LDL-C) particle. It has been shown that AIP is a strong marker to predict the risk of atherosclerosis and coronary heart disease [8-10]. Considering of the close relationship between the dyslipidemia and NAFLD, we assume that AIP may be a maker to predict NAFLD, especially in obese people.

The aim of our research is to figure out whether AIP can be a marker to predict the diagnosis of NAFLD in obese people.

\section{Methods}

\section{Study population}

All the eligible participants were over 18 years old and had been living in their current residence for at least 5 years from each community or village. Inclusion criteria were as follows: (1) age $\geq 18$ years, (2) BMI $\geq 28 \mathrm{~kg} / \mathrm{m} 2$. Exclusion criteria were as follows: (1) missing important information (such as age, sex or ultrasonography), (2) complications or conditions that affected lipid metabolism, (3) taking any drugs that lipid metabolism in the previous three months, and (4) obviously poor compliance. All of them signed informed consent before the examination.

\section{Data collection}

Data was collected at local health stations by trained medical staff. Weight was measured in kilograms while height was measured in centimeters, respectively. Waist circumference (WC) was measured at the umbilicus level with the participants in the standing position. BMI was equal to weight $(\mathrm{kg})$ divided by squared height $(\mathrm{m})$.

Blood samples were collected from all participants after an overnight fast of at least $10 \mathrm{~h}$. The serum lipid parameters, glutamyl transpeptidase (GGT), aspartate aminotransferase (AST), alanine aminotransferase (ALT) and fast plasma glucose (FPG) were measured with the ARCHITECT ci16200 Integrated System (Abbott, Illinois, USA).

\section{Definitions of NAFLD, AIP stratification and obesity}

Diagnosis of fatty liver by ultrasonography is defined by the presence of at least two of three abnormal findings: diffusely increased echogenicity ('bright') liver - with stronger echoes than in the renal parenchyma, vascular blurring, and narrowing of the lumen of the hepatic veins [9]. In addition, other liver diseases should be excluded [11]. According to previous studies, AIP was stratified into three groups: low- $(<0.11)$, intermediate$(0.11-0.21)$ and high-risk $(>0.21)[9,12,13]$. Overweight was defined as a BMI of 24.0 to 27.9 , and obesity was defined as a BMI of 28.0 or higher [14].

\section{Data analysis}

Gender, age, BMI, WC, total cholesterol (TC), TG, LDL-C, FPG, HDL-C was showed in different groups according to NAFLD or not. Differences between mean values were tested using Student's t-test. The comparison among groups in Table 1 was determined by one-way analysis of variance (ANOVA).Chi-squared test was used to analyze whether if there were any statistically difference in the prevalence of NAFLD in different AIP quartiles. Logistic regression models were used to analyze the association between AIP and NAFLD. Odds ratios (ORs) were obtained from logistic regression analysis, and the results were presented as ORs with a $95 \%$ confidence interval (CI). A $P$ value of $<0.05$ was defined as statistically significant. The accuracy of using AIP and other related parameters to predict NAFLD was assessed by calculating the non-parametric area under the receiver-operating characteristic (ROC) curve (AUC) with 95\% CI. With the limitation of detection means, the criteria of NAFLD used in our research was by ultrasound.

\section{Results}

A total of 538 obese people were included in the study. Table 1 described the characteristics of the participants. The mean age was $42.18 \pm 11.59$ years, and 281 (52.23\%)

Table 1 Clinical characteristics in three groups according to AIP levels

\begin{tabular}{lllll}
\hline & low & intermediate & high & $P$ \\
\hline Male (\%) & 34.0 & 63.4 & 69.9 & 0.000 \\
Age & $42.6 \pm 11.8$ & $42.2 \pm 11.2$ & $41.7 \pm 11.5$ & 0.718 \\
BMl & $30.48 \pm 2.16$ & $30.63 \pm 2.03$ & $30.97 \pm 2.21$ & 0.058 \\
WC & $96.45 \pm 8.75$ & $99.22 \pm 10.76$ & $100.26 \pm 8.68$ & 0.000 \\
AIP & $-0.11 \pm 0.15$ & $0.16 \pm 0.03$ & $0.48 \pm 0.28$ & 0.000 \\
TC & $5.04 \pm 0.93$ & $5.35 \pm 1.04$ & $5.45 \pm 1.12$ & 0.000 \\
LDL & $2.78 \pm 0.70$ & $3.13 \pm 0.74$ & $3.07 \pm 0.74$ & 0.000 \\
HDL & $1.25 \pm 0.22$ & $1.09 \pm 0.19$ & $0.92 \pm 0.21$ & 0.000 \\
TG & $1.00 \pm 0.28$ & $1.57 \pm 0.27$ & $3.16 \pm 2.20$ & 0.000 \\
ALT & $19.82 \pm 11.43$ & $38.17 \pm 95.01$ & $29.38 \pm 39.73$ & 0.000 \\
AST & $24.58 \pm 6.96$ & $41.87 \pm 122.09$ & $28.82 \pm 8.72$ & 0.019 \\
GGT & $28.94 \pm 21.33$ & $40.24 \pm 29.03$ & $63.64 \pm 71.70$ & 0.000 \\
FPG & $5.76 \pm 1.33$ & $5.89 \pm 1.59$ & $6.07 \pm 1.72$ & 0.110 \\
\hline
\end{tabular}




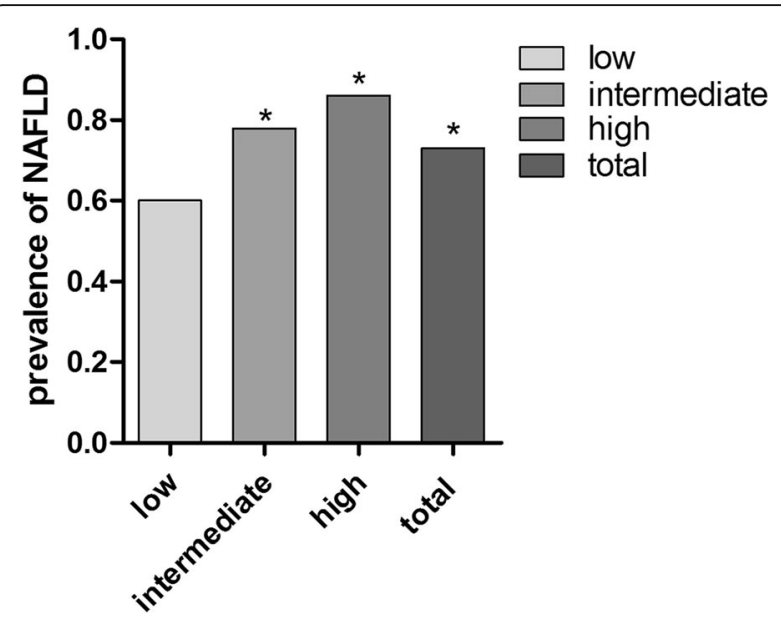

Fig. 1 the prevalence of NAFLD according to AIP stratification Lengends: AIP was classified into three levels: low $(<0.11)$, intermediate $(0.11-0.21)$, high $(>0.21)$ risk. * means significant difference exists compared to low group

were male. The parameters were also described in groups according to AIP stratification: low $(n=250$, $46.5 \%)$, intermediate $(n=82,15.2 \%)$, high $(n=206$, 38.3\%). Significant differences were observed in lipid profile, WC, AST, ALT, GGT grouped by AIP. The prevalence of NAFLD was increased in intermediate group and high group. There are significant differences between the low group and other groups in Fig. 1. Figure 2 showed the levels of TG, HDL-C and AIP according to the diagnosis of NAFLD grouped by gender. Significant discrepancy showed in all the three parameters in both genders.

Table 2 shows the association between the anthropometric parameters with NAFLD. When including AIP, TC, LDL-C, ALT, AST, GGT, FPG, gender, age, WC, $\mathrm{BMI}$ in the multivariate logistic regression analysis of NAFLD, AIP, TC, LDL-C, GGT, WC, BMI showed a significant relationship with NAFLD. Among these, AIP showed a strong relationship (5.37, 2.26-12.73, $p<$ 0.000 ) with NAFLD.

To assess the accuracy of anthropometric parameters to predict the diagnosis of NAFLD, AUC were conducted which were showd in Table 3. Among all the anthropometric parameters, AIP showed to be the best predictor with an AUC of 0.718 in the obese participants we included. Gender difference can be seen from the table. The results in men fitted those of the total participants. However, AUC of AIP was lower than GGT, BMI and $\mathrm{WC}$ in female.

\section{Discussion}

There are 3 main finding in the study. First, this research demonstrated that there were concordances between increased AIP and significant increase in the value of BMI, WC, ALT, GGT and lipid profile (Table 1). This finding is similar with the previous study about the association between AIP and cardiovascular diseases. Second, AIP with some other parameters were strongly associated with NAFLD in the multivariate logistic regression. Third, AIP was the best predictor of NAFLD with an AUC of 0.718 compared with other related indexes in the total participants. But in female participants, GGT turned out to be the best $(\mathrm{AUC}=0.689)$ in all the parameters.

A large population-based study found that $91 \%$ of obese individuals $\left(\mathrm{BMI}>30 \mathrm{~kg} / \mathrm{m}^{2}\right.$ ) had evidence of steatosis on ultrasound [15]. The obese participants in our research were all BMI $\geq 28 \mathrm{~kg} / \mathrm{m}^{2}$. The prevalence of NAFLD in our study was $73 \%$, which means that about a quarter of obese people had no fatty liver. Although BMI is one of the most common measurement to categorize overweight or obesity, increasing evidence suggests that BMI is not a precise measure of body fat related risk of non-communicable diseases. Another study also showed AIP to be an important tool in obese participants with cardiovascular disease. More measurements like visceral fat area, inflammatory

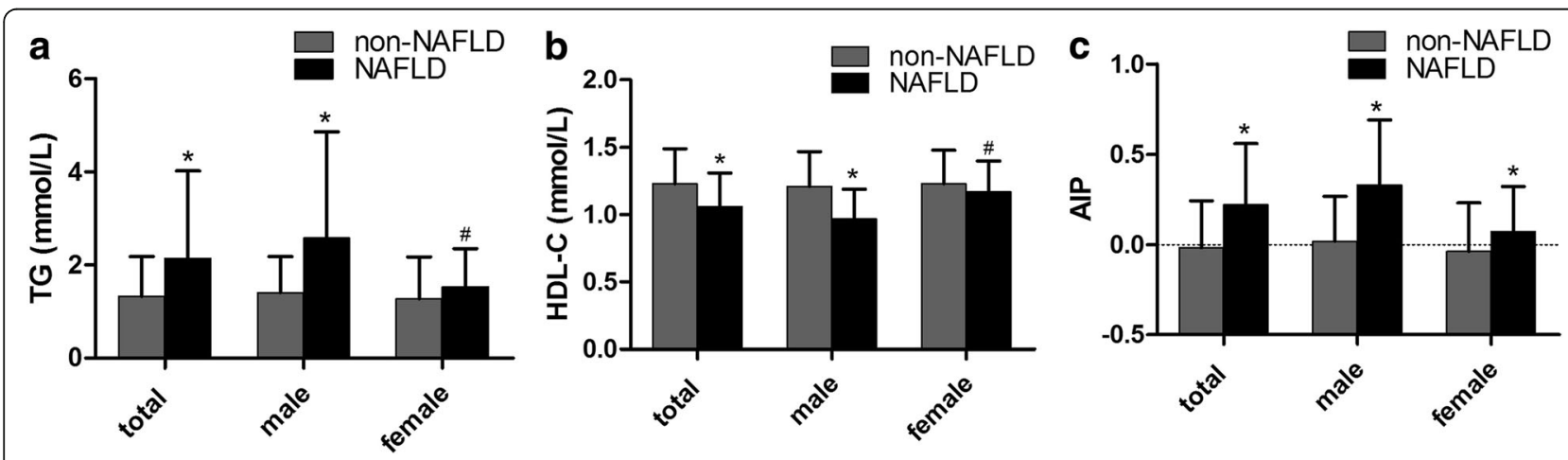

Fig. 2 TG, HDL-C and AIP levels according to NAFLD (non-NAFLD participants vs NAFLD participants) in different groups by genderLegends: a. TG (mmol/L); b. HDL-C (mmol/L); c. AIP. Significance levels: * $<0.001$, \#<0.05. 
Table 2 Multivariable logistic regression analyses on the association between AIP and NAFLD

\begin{tabular}{llll}
\hline & OR & $95 \% \mathrm{Cl}$ & $\mathrm{P}$ \\
\hline AIP & 5.37 & $2.26-12.73$ & 0.000 \\
TC & 0.47 & $0.24-0.95$ & 0.036 \\
LDL & 3.04 & $1.18-7.85$ & 0.021 \\
ALT & 1.04 & $1.00-1.08$ & 0.027 \\
AST & 0.97 & $0.94-1.00$ & 0.023 \\
GGT & 2.10 & $1.20-3.67$ & 0.009 \\
FPG & 0.99 & $0.85-1.14$ & 0.842 \\
BMI & 1.27 & $1.11-1.46$ & 0.001 \\
WC & 1.05 & $1.01-1.08$ & 0.005 \\
GENDER & 0.59 & $0.33-1.05$ & 0.070 \\
AGE & 0.99 & $0.97-1.01$ & 0.432 \\
\hline
\end{tabular}

factors were included in the predictor of NAFLD. But almost all of them were not commonly used in clinical practice. An accurate index is needed to screen and predict NAFLD in clinical applications.

The progression of NAFLD results from an imbalance between lipid uptake and lipid disposal and eventually causes oxidative stress and hepatocyte injury. Although the precise pathological process has not been elucidated, it is been widely accepted that excess TG accumulation in the liver is the prerequisite for NAFLD. TG showed a great correlation with NAFLD in both epidemiologic studies [15] and animal research [16] . HDL-C was significantly decreased in participants with NAFLD in our research (Fig. 2). With the close correlation between TG, HDL-C and NAFLD, we assumed that an index which was calculated with TG and HDL-C may be a predictor for NAFLD. AIP was calculated as log (TG/ HDL-C). AIP was used in some research to predict cardiovascular diseases and metabolic syndrome. With being logarithmically transformed, TG/HDL-C could be adjusted for the lack of normative distribution and demonstrate a correlation with smaller LDL-C particles. Compared with the traditional lipid profile, AIP was proved to be a better predictor of atherosclerosis than LDL-C $[13,17]$.

When we compared some related parameters of NAFLD, AIP showed a better AUC in predicting NAFLD than any other indexes. Previous studies have shown that higher TG/HDL-C indicates insulin resistance, which could be another possible explanation for AIP being a good index considering that insulin resistance is closely related with NAFLD. Another study were in line with our study which were also conducted in obese participants. It was reported that patients with prominent obesity, but with low TG/HDL-C values, had only a low increased risk of coronary heart disease or cardiovascular disease, while patients with high TG/HDL-C had a $70 \%$ increased risk, compared to normal weight and low TG/HDL-C as reference. The AUC of WC (0.695) was larger than that of BMI (0.671), which was consistent with another study on the strategies for predicting NAFLD [18]. This result may be explained by the fact that Asians are more likely to have central fat deposition even in a lower BMI. Although GGT seemed to be a good predictor $(A U C=0.703)$ in our research, few research paid attention on it. It is more related with NAFLD on its severity.

Table 3 showed gender difference in the AUC of AIP for the presence of NAFLD. AIP seemed to be a better predictor in male subjects. The difference was also seen in other studies of NAFLD, which TG and HDL-C showed significant differences for NAFLD in men, while not in women $[19,20]$. These results differ from the previous studies from India, in which TG showed a better index in female for NAFLD [21]. These relationships

Table 3 The area under the receiver operating characteristic curves of AIP, lipid profiles, AST, ALT, GGT, FPG, BMI, WC for the presence of NAFLD classified by gender

\begin{tabular}{|c|c|c|c|c|c|c|c|c|c|}
\hline & \multicolumn{3}{|l|}{ Total } & \multicolumn{3}{|l|}{ Male } & \multicolumn{3}{|l|}{ Female } \\
\hline & AUC OR & $95 \% \mathrm{Cl}$ & $P$ & AUC OR & $95 \% \mathrm{Cl}$ & $P$ & AUC OR & $95 \% \mathrm{Cl}$ & $P$ \\
\hline AIP & 0.718 & $0.670-0.766$ & 0.000 & 0.775 & $0.709-0.841$ & 0.000 & 0.633 & $0.561-0.706$ & 0.000 \\
\hline GGT & 0.703 & $0.653-0.753$ & 0.000 & 0.675 & $0.598-0.752$ & 0.000 & 0.689 & $0.620-0.759$ & 0.000 \\
\hline $\mathrm{TC}$ & 0.577 & $0.522-0.631$ & 0.006 & 0.547 & $0.459-0.634$ & 0.282 & 0.588 & $0.515-0.660$ & 0.020 \\
\hline $\mathrm{LDL}$ & 0.607 & $0.552-0.661$ & 0.000 & 0.570 & $0.482-0.658$ & 0.106 & 0.615 & $0.544-0.687$ & 0.002 \\
\hline $\mathrm{HDL}$ & 0.309 & $0.259-0.358$ & 0.000 & 0.239 & $0.166-0.313$ & 0.000 & 0.420 & $0.347-0.493$ & 0.034 \\
\hline $\mathrm{TG}$ & 0.698 & $0.649-0.748$ & 0.000 & 0.731 & $0.658-0.803$ & 0.000 & 0.633 & $0.561-0.704$ & 0.000 \\
\hline ALT & 0.679 & $0.630-0.728$ & 0.000 & 0.640 & $0.559-0.721$ & 0.001 & 0.635 & $0.567-0.704$ & 0.000 \\
\hline AST & 0.617 & $0.566-0.668$ & 0.000 & 0.571 & $0.490-0.652$ & 0.099 & 0.575 & $0.505-0.646$ & 0.046 \\
\hline FPG & 0.596 & $0.544-0.648$ & 0.001 & 0.600 & $0.512-0.689$ & 0.045 & 0.549 & $0.478-0.619$ & 0.197 \\
\hline $\mathrm{BMI}$ & 0.671 & $0.622-0.720$ & 0.000 & 0.728 & $0.655-0.800$ & 0.000 & 0.639 & $0.571-0.707$ & 0.000 \\
\hline WC & 0.695 & $0.647-0.743$ & 0.000 & 0.683 & $0.609-0.757$ & 0.000 & 0.658 & $0.590-0.726$ & 0.000 \\
\hline
\end{tabular}


may partly be explained by the effect of different adipose tissue distribution according to gender which played important role in the development of NAFLD. Though subcutaneous adipose tissue is similar in both gender with NAFLD but male store more visceral adipose tissue compared to female, which means BMI could be a rather inaccurate for male subjects. This also fitted with our results that the AUC of BMI is higher than that of AIP in female participants. Another possible explanation for the gender difference may be related with the effect of varying sex hormone on NAFLD [22].

\section{Limitation}

First this was a cross-sectional study and could not definitively provide a causal relationship between AIP and NAFLD. Second, no grades on NAFLD were included in our study. In the future more research should focus on the severity of NAFLD. Third, potential confounders, especially those could affect NAFLD, such as lifestyle and dietary habits, were not included in our study. Last, the diagnosis of NAFLD was not through biopsy but through ultrasonography. However, in the Chinese guideline and most guidelines in other countries, liver biopsy is not recommended but also not applicable with high sensitivity and specificity in all the participants. Ultrasonography is the most applicable and convenient way to diagnosis NAFLD [23].

\section{Conclusion}

In summary, our study showed that AIP were strongly correlated with NAFLD in obese participants. AIP should be used as a regular monitoring index of NAFLD in clinical practice, especially for obese men.

\section{Abbreviations}

AIP: atherogenic index of plasma; ALT: alanine aminotransferase; ANOVA: analysis of variance; AST: aspartate aminotransferase; AUC: nonparametric area under the receiver-operating characteristic curve; BMI: body mass index; Cl: confidence interval; FPG: fast plasm glucose; GGT: glutamyl transpeptidase; HDL-C: high density lipoprotein cholesterol; LDL-C: Iow density lipoprotein cholesterol; NAFLD: non-alcoholic fatty liver disease; OR: odds ratios; ROC: receiver-operating characteristic; TC: total cholestrol; TG: triglyceride; WC: waist circumference

\section{Acknowledgements}

Not applicable.

\section{Funding}

This work was supported by grants from National Key Research and Development Program of China (2017YFC1309800 and 2017YFC0909600), Key Research and Department of Shandong Province (2016GSF201013), Key Research and Department of Shandong Province (2016GSF201015), The Clinical Research Fund of Chinese Medical Association (15010010589), The Research Fund for Public Welfare, National Health and Family Planning (201402005), Key Research and Department of Shandong Province (2017GSF18184), National Major Scientific and Technological Special Project for "Significant New Drugs Development" (2015ZX09101019).

\section{Availability of data and materials}

All data generated or analyzed during this study are included in this published article.

\section{Authors' contributions}

Study conception and design: Q.W. and DM.Z.. Literature search: Q.W., L.F., and DM.Z. Data collection and analysis: Q.W., J.L. and DM.Z. Data interpretation: L.F. and DM.Z. Writing: Q.W. and Q.L. All authors read and approved the final manuscript.

\section{Authors' information}

Not applicable.

\section{Ethics approval and consent to participate}

The study protocol was approved by the ethics committee of Shanghai Jiao Tong University Written informed consent was obtained from all participants following a detailed description of the purpose of the study.

\section{Consent for publication}

Written informed consent for publication of their clinical details was obtained from the patients. Copies of the consent form are available for review by the Editor of this journal.

\section{Competing interests}

The authors declare that they have no competing interests.

\section{Publisher's Note}

Springer Nature remains neutral with regard to jurisdictional claims in published maps and institutional affiliations.

\section{Author details}

'Department of Endocrinology, Shandong Provincial Hospital affiliated to Shandong University, 324 Jing 5 Road, Jinan 250021, Shandong Province, China. ${ }^{2}$ Shandong Clinical Medical Center of Endocrinology and Metabolism, 324 Jing 5 Road, Jinan 250021, Shandong Province, China. Institute of Endocrinology and Metabolism, Shandong Academy of Clinical Medicine, 324 Jing 5 Road, Jinan 250021, Shandong Province, China. ${ }^{4}$ Department of Ultrasound, Shandong Provincial Hospital affiliated to Shandong University, 324 Jing 5 Road, Jinan 250021, Shandong Province, China.

Received: 9 April 2018 Accepted: 27 November 2018

Published online: 13 December 2018

\section{References}

1. Younossi ZM, Koenig AB, Abdelatif D, Fazel Y, Henry L, Wymer M. Global epidemiology of nonalcoholic fatty liver disease-meta-analytic assessment of prevalence, incidence, and outcomes. Hepatology. 2016;64(1):73-84.

2. Loomba R, Sanyal AJ. The global NAFLD epidemic. Nat Rev Gastroenterol Hepatol. 2013;10(11):686-90.

3. Liu TT, Zhou XT, Li WL, Peng YY, Liu XJ, Wang J, et al. Prevalence of Overweight and Obesity in Adult Populations in Mianyang, Sichuan Province. Sichuan da xue xue bao Yi xue ban = Journal of Sichuan University Medical science edition. 2017;48(6):946-8.

4. Ogden CL, Fakhouri TH, Carroll MD, Hales CM, Fryar CD, Li X, et al. Prevalence of obesity among adults, by household income and education - United States, 2011-2014. MMWR Morb Mortal Wkly Rep. 2017;66(50):1369-73.

5. Malik VS, Willett WC, Hu FB. Global obesity: trends, risk factors and policy implications. Nat Rev Endocrinol. 2013;9(1):13-27.

6. Bhaskaran K, Douglas I, Forbes H, dos-Santos-Silva I, Leon DA, Smeeth L. Bodymass index and risk of 22 specific cancers: a population-based cohort study of 5.24 million UK adults. Lancet (London, England). 2014;384(9945):755-65.

7. Lane M, Zander-Fox DL, Robker RL, McPherson NO. Peri-conception parental obesity, reproductive health, and transgenerational impacts. Trends in endocrinology and metabolism. 2015;26(2):84-90.

8. Nwagha UI, Ikekpeazu EJ, Ejezie FE, Neboh EE, Maduka IC. Atherogenic index of plasma as useful predictor of cardiovascular risk among postmenopausal women in Enugu, Nigeria. African health sciences. 2010;10(3):248-52.

9. Shen S, Lu Y, Qi H, Li F, Shen Z, Wu L, et al. Association between ideal cardiovascular health and the atherogenic index of plasma. Medicine. 2016; 95(24):e3866.

10. Chang Y, Li Y, Guo X, Dai D, Sun Y. The Association of Ideal Cardiovascular Health and Atherogenic Index of plasma in rural population: a cross-sectional study from Northeast China. Int J Environ Res Public Health. 2016;13(10):1027. 
11. [Diagnostic criteria of nonalcoholic fatty liver disease]. Zhonghua gan zang bing za zhi = Zhonghua ganzangbing zazhi $=$ Chinese journal of hepatology 2003;11(2):71.

12. Akbas EM, Timuroglu A, Ozcicek A, Ozcicek F, Demirtas L, Gungor A, et al. Association of uric acid, atherogenic index of plasma and albuminuria in diabetes mellitus. Int J Clin Exp Med. 2014;7(12):5737-43.

13. Dobiasova M, Frohlich J. The plasma parameter log (TG/HDL-C) as an atherogenic index: correlation with lipoprotein particle size and esterification rate in apoB-lipoprotein-depleted plasma (FER (HDL)). Clin Biochem. 2001;34(7):583-8.

14. society Cd. China guideline for type 2 Diabetes 2010 .

15. Amarapurkar D, Kamani P, Patel N, Gupte P, Kumar P, Agal S, et al. Prevalence of non-alcoholic fatty liver disease: population based study. Ann Hepatol. 2007;6(3):161-3.

16. Samuel VT, Liu ZX, Qu X, Elder BD, Bilz S, Befroy D, et al. Mechanism of hepatic insulin resistance in non-alcoholic fatty liver disease. J Biol Chem. 2004;279(31):32345-53.

17. Dobiasova M, Frohlich J. The new atherogenic plasma index reflects the triglyceride and HDL-cholesterol ratio, the lipoprotein particle size and the cholesterol esterification rate: changes during lipanor therapy. Vnitrni lekarstvi. 2000;46(3):152-6.

18. Lee $\mathrm{JH}$, Jeong $\mathrm{SJ}$. What is the appropriate strategy for diagnosing NAFLD using ultrasonography in obese children. World journal of pediatrics. 2017; 13(3):248-54.

19. Lonardo A, Trande P. Are there any sex differences in fatty liver? A study of glucose metabolism and body fat distribution. J Gastroenterol Hepatol. 2000;15(7):775-82.

20. Hossain IA, Akter S, Rahman MK, Ali L. Gender specific Association of Serum Leptin and Insulinemic Indices with nonalcoholic fatty liver disease in Prediabetic subjects. PLoS One. 2015;10(11):e0142165.

21. Srinivas M, Srinivasan V, Mohan MB, Varghese J. Venkataraman J. a study of gender-wise risk association between fatty liver and metabolic syndrome components (Asia-Pacific criteria) in a south Indian urban cohort. Indian journal of gastroenterology. 2015;34(1):38-42.

22. Pan JJ, Fallon MB. Gender and racial differences in nonalcoholic fatty liver disease. World J Hepatol. 2014;6(5):274-83.

23. Association FlaaldsgotCM. Guidelines for the diagnosis and treatment of nonalcoholic fatty liver disease. Chinese Journal of Internal Medicine. 2010;6(5):9.

Ready to submit your research? Choose BMC and benefit from:

- fast, convenient online submission

- thorough peer review by experienced researchers in your field

- rapid publication on acceptance

- support for research data, including large and complex data types

- gold Open Access which fosters wider collaboration and increased citations

- maximum visibility for your research: over $100 \mathrm{M}$ website views per year

At $\mathrm{BMC}$, research is always in progress.

Learn more biomedcentral.com/submissions 